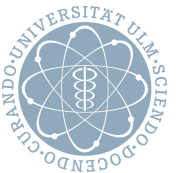

\title{
Adaptive Clustering for Contour Estimation of Vehicles for High-Resolution Radar
}

Johannes Schlichenmaier, Fabian Roos, Martin Kunert, and Christian Waldschmidt

(C) 2016 IEEE. Personal use of this material is permitted. Permission from IEEE must be obtained for all other uses, in any current or future media, including reprinting/republishing this material for advertising or promotional purposes, creating new collective works, for resale or redistribution to servers or lists, or reuse of any copyrighted component of this work in other works.

DOI: 10.1109/ICMIM.2016.7533930 


\title{
Adaptive Clustering for Contour Estimation of Vehicles for High-Resolution Radar
}

\author{
Johannes Schlichenmaier*, Fabian Roos*, Martin Kunert ${ }^{\dagger}$, and Christian Waldschmidt* \\ ${ }^{*}$ Institute of Microwave Engineering, Ulm University, 89081 Ulm, Germany \\ Email: johannes.schlichenmaier@uni-ulm.de \\ ${ }^{\dagger}$ Robert Bosch GmbH, Advanced Engineering Sensor Systems, P.O. Box 16 61, 71226 Leonberg, Germany
}

\begin{abstract}
Future automotive radars will be able to achieve much higher range and angular resolution compared to currently used radar sensors. This enables functionalities like vehicle contour estimation to be used in advanced driver assistance systems, thus heavily increasing their performance. In this paper, the application of an adaptive algorithm on basis of k-nearestneighbours examination for clustering radar data as precursor to estimation of width, length, and position of vehicles is presented and compared to a more basic algorithm. The influence of the parameters of this KNN-DBSCAN algorithm and its performance dependency on the used MIMO radar system is discussed.
\end{abstract}

\section{INTRODUCTION}

Today's driver assistance and autonomous driving systems use a combination of multiple sensors to estimate the surroundings of the ego-car and deliver useful functionality, ranging from warning the driver of dangerous situations, initiating emergency systems and maneuvers, up to planning and adjusting their driving trajectory autonomously.

As of now, radar based data is primarily used for detecting the range, angle, and velocity of medium sized objects like cars as a whole, rather than trying to gain information about their dimension or shape. This limitation stems from the low resolution partly in range and especially in angular direction.

Currently, radar systems are being developed, which by far exceed the limitations of current systems with regard to range and angular resolution. This means that objects detected by radar are not bound to appear as a single target anymore, but instead behave like extended objects, resulting in multiple reflection points per object, even if their size is not overly large [1]. This makes contour estimation systems based on radar imagery a viable option in place of or in addition to optical systems like LiDAR or video systems. Radar is especially attractive because of the known advantages toward optical systems like higher robustness in harsh weather conditions such as fog, snow, or rain, as well as providing its own illumination source. With radar it is even possible to detect targets in visibility shadows of other objects via ground reflections.

Up to this time, only a few investigations on the topic of radar based contour estimation exist. In [2] an algorithm chain for obtaining an oriented bounding box based upon a density based clustering algorithm [3] is presented.

The algorithm chain in this paper is based upon the approach in [2], but uses an adaptive clustering algorithm presented in [4]. This $K N N-D B S C A N$ algorithm is better equipped for self-configuration and adaptation to different radar data characteristics with respect to reflection point distribution and density.

The focus lies on parameter discussion of the involved algorithms and the radar system in regard to contour estimation.

\section{Problem Formulation}

As shown in [2], clustering is a vital part on the way to reliably obtain contours from radar data. An ideal clustering algorithm is able to correctly lump together all those reflection points belonging to one object, regardless of how dense these points are, without adding any neighbouring noise points that may corrupt the resulting contour estimation. If too many noise points are included in a cluster, it may lead to an overestimation of the contour in the later steps of the algorithm chain. If the algorithm cannot handle spread out reflex points, it may lead to an underestimation, because not enough available information will be used in the later steps, up to a point where maybe no contour can be detected at all. It can be argued that overestimation is less critical than underestimation with regards to traffic safety.

\section{Assessment CRITERIA}

To evaluate the clustering performance in regard to the chosen parameters, the clusters acquired in the evaluated measurements are examined with respect to the following requirements:

1) A valid cluster has to intersect with the known ground truth of the object and may not exceed a length of $8 \mathrm{~m}$ and a width of $3 \mathrm{~m}$.

2) The more the cluster area is intersecting with the ground truth, the higher the value of the cluster. This is called overlap and is at its maximum, if the whole ground truth is covered by the cluster.

3) The more the cluster area lies inside the ground truth instead of the outside, the higher the value of the cluster. This is called confinement and is at its maximum, if the whole cluster is situated within the boundaries of the ground truth.

4) To determine a score for each parameter set, the above criteria are combined summing up the multiplication of overlap and confinement values for every valid cluster found in the measurements. 


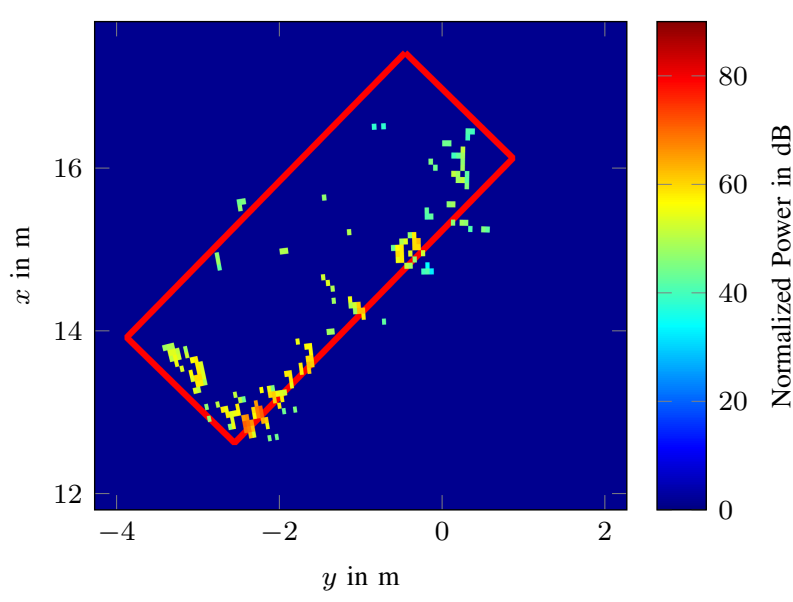

(a) Scenario A: favorable reflection point distribution.

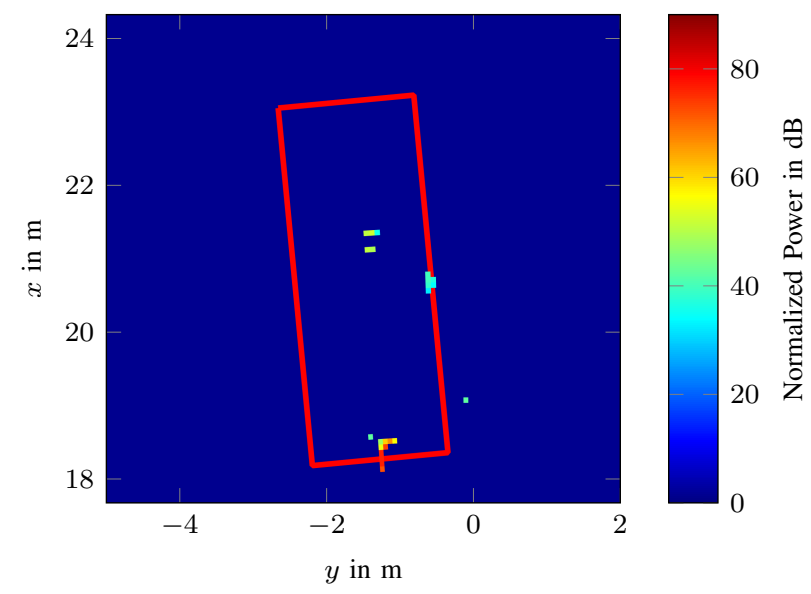

(b) Scenario B: challenging reflection point distribution.

Fig. 1. Radar measurements of two automotive scenarios; acquired with a 2x4 MIMO system using a bandwidth of 2 GHz; ground truth as red rectangle.

\section{RADAR DATA AS BASIS FOR CONTOUR ESTIMATION}

There is already a substantial amount of research done regarding contour estimation based on optical systems like video cameras and LiDAR [5]-[7]. Image data from optical systems often yields a high number of accurate measurement points along the contour of objects. In LiDAR, this stems from the mechanically panning of a highly focused laser beam and in video images from high resolution CMOS sensors and the usage of perceived color and brightness as the main means to distinguish separate parts in the image.

For radar, these prerequisites are not given. For most practical automotive applications, the antenna beam width of the radar system is not as focused and angular information is acquired by means of direction of arrival estimation introducing a not negligible factor of uncertainty and spread of targets. Furthermore, the longer wavelength leads to a highly unforeseeable amount of interactions of radar signals with the object and its surroundings, depending on properties like distance, incident angle, roughness, volume, shape, etc. In combination with the possibility of multiple reflections under the body of the object or in its cavities and at the ground around it (clutter), this leads to the familiar 2D radar image exhibiting highly scattered reflection points in, along, and around the shape of the object.

The radar data used to test the clustering performance of the algorithms was obtained from a next generation radar with 2 transmitting and 4 receiving channels operating in Time Division Multiplex (TDM) mode to obtain $2 \times 4$ MIMO capability. A bandwidth of $B=2 \mathrm{GHz}$ around a center frequency of $f_{\mathrm{c}}=76.45 \mathrm{GHz}$ was used to obtain a range resolution of $\Delta r=7.5 \mathrm{~cm}$. The radar was operated in chirp-sequence modulation, using $N_{\mathrm{r}}=128 \mathrm{ramps}$, with $N_{\mathrm{s}}=1024$ samples at a sampling rate of $f_{\mathrm{s}}=10 \mathrm{MHz}$. This leads to a velocity resolution of $\Delta v=5.4 \mathrm{~cm} / \mathrm{s}$ with a maximum unambiguous velocity of $v_{\max }=3.43 \mathrm{~m} / \mathrm{s}$. Target extraction was done via a combination of OS-CFAR algorithms in both range and velocity direction. Both CFAR thresholds were chosen to be relatively low to gather as much extended targets as possible. For direction of arrival (DOA) estimation, a maximum likelihood estimator was used to compare the phase relation of the extracted targets between the 8 MIMO channels against previously acquired single target calibration data.

For obtaining ground truth data, a Mercedes-Benz E-Class Model $\mathrm{T}$ equipped with an Automotive Dynamic Motion Analyzer (ADMA) sensor unit was used to accurately measure the differential GPS coordinates at any given time during the measurement. The car performed a series of maneuvers such as left and right turning, as well as stopping and driving in reverse. For the parameter discussion, only measurements where the velocity relative to the sensor was within the unambiguous velocity range were considered.

In Fig. 1 two exemplary measurement scenarios are shown. The known ground truth is depicted as a red rectangle. Figure 1 (a) shows a very favorable measurement scenario. The car is currently executing a turning motion, which leads to more reflection surfaces for the radar signal and more velocity contributions that qualify as targets. In Fig. 1 (b) the car is not moving as well as facing the radar with its short side at an inappropriate angle leading to very little interaction.

\section{Algorithm CHAin}

After target extraction, the coordinates of said targets are fed into the algorithm depicted in Fig. 2 chain in which the clustered data is used to construct a bounding box describing the estimated position, width, length, and yaw angle of the vehicle.

\section{A. KNN-DBSCAN Clustering}

For the clustering, firstly an examination of the smoothed probability density function of the distance distribution between every point is performed. In the original presentation of the algorithm in [4] a distinct multi-waveform is assumed for the density. The distance value of the first local minimum is used for the so-called window width. This value is used in the following steps to build the fuzzy clusters, on which 


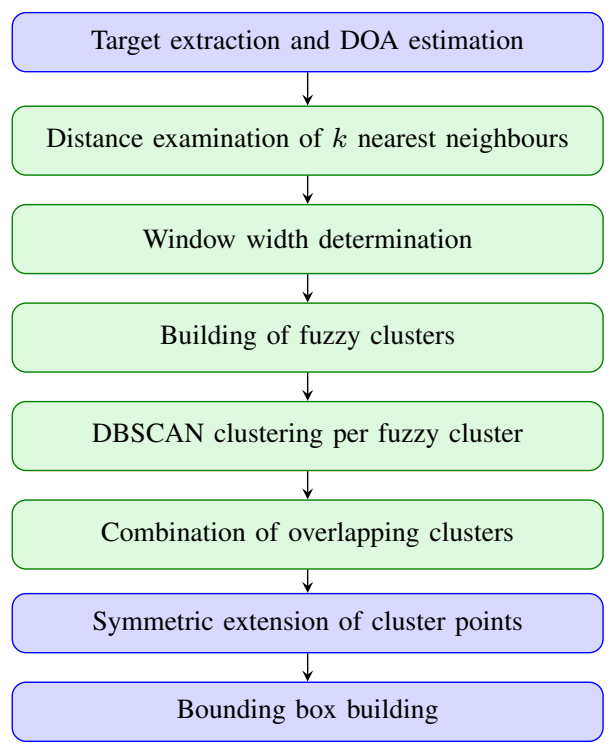

Fig. 2. Algorithm chain for contour estimation with KNN-DBSCAN.

a DBSCAN clustering, as described in [8], is subsequently performed. The parameters $\epsilon$ and $p_{\min }$ are calculated for every fuzzy cluster separately from the window widths of the points in the cluster as described in [8]. Overlapping result clusters are then combined.

In Fig. 3 the smoothed distance distribution for two exemplary target points situated within the boundaries of the ground truth of Fig. 1 (a), denoted by A.1 and A.2, is plotted for two different smoothing factors $\alpha$. Target points belonging to the vehicle often exhibit a density spectrum, in which the first wave is not sharply enough separated from the next wave when using the default smoothing factor. This leads to too large clusters often incorporating lots of noise points into the fuzzy cluster (and subsequently the final cluster). If the smoothing is sufficiently reduced, so that the distance distribution of point $A .1$ exhibits the desired behaviour, distributions like that of point A.2 experiences a high fluctuation leading to too small clusters. Therefore, it was decided to set the criteria for the window width to the first local maximum instead, which leads to a more stable behaviour in regards to the smoothing. It naturally leads to slightly smaller than optimal clusters, which are usually automatically combined in the last step of clustering.

\section{B. Bounding Box Construction}

After clustering a simple method for a rough estimation of width, length, and position is used. This method consists of a symmetric extension (point reflection of targets on the geometrical center of the cluster) of the cluster points and a subsequent construction of the bounding box of minimal area including all points of the cluster. To avoid over-enlargement of the bounding box due to points situated on the far side of the object (relative to the sensor), which often stem from multi-reflection paths, only the points on the near side of the object are considered for a symmetric extension.

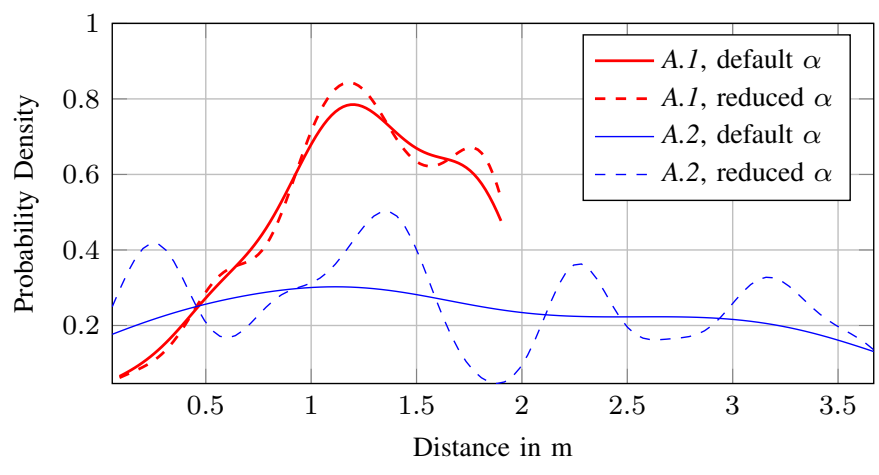

Fig. 3. Kernel-smoothed distribution of distances to the 100 nearest neighbours for two sample points $A .1$ and $A .2$ from the favorable measurement scenario A for different smoothing factors $\alpha$.

\section{PARAMETER Discussion}

\section{A. KNN-DBSCAN Parameters}

When examining the $K N N-D B S C A N$ algorithm, the calculation of the window width is critical, because creation of the fuzzy clusters and the calculation of parameters $\epsilon$ and $p_{\text {min }}$ heavily rely on this step. The integral part of this step is the calculation of the distance probability distribution. Three parameters are identified to strongly influence the shape of the distribution:

\section{1) Kernel function $\mathscr{K}$}

The kernel describes the function, the distance histogram is convoluted with in order to obtain the kernel smoothed distance probability distribution. Four kernel functions are examined:

- Gaussian kernel

- Rectangular kernel

- Triangular kernel

- Epanechnikov kernel

The Epanechnikov kernel describes a hill-like curve with edges abruptly assuming zero instead of approaching it like a Gaussian function.

2) Kernel smoothing factor $\alpha$

The smoothing factor of the kernel determines the amount of smoothing the histogram experiences during the convolution and is unitless. After some experimentation, it emerged that values below 0.5 often lead to too fluctuating distributions. Similarly, values above 2.0 almost always result in uselessly large clusters in the presence of noise and clutter. Therefore, values of $\alpha=0.5 . .2 .0$ were examined.

3) Number of nearest neighbours $k$

On the one hand, the number of nearest neighbours $k$ to take into consideration during calculating the distance distribution should not be too low, since otherwise the first wave might not be yet distinct enough to determine a sensible window width. On the other hand, $k$ should not be too large, as it greatly influences the calculation time. Here, a parameter sweep of $k=50 . .200$ nearest neighbours was performed. 


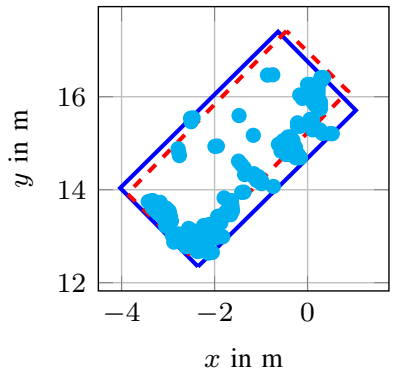

(a) Scenario A: favorable

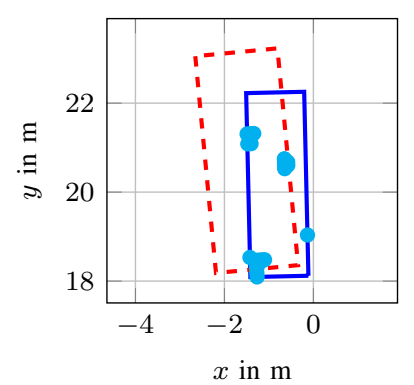

(b) Scenario B: challenging
Fig. 4. KNN-DBSCAN clustering result (๑) for optimal parameter choice, estimated contours ( $(-)$ with ground truth $(-=-)$. DBSCAN achieved the same result for Scenario A, but failed to detect any cluster in Scenario B.

\section{B. DBSCAN Parameters}

To compare performance of $K N N-D B S C A N$ with that of basic $D B S C A N$, a range of $\epsilon=0.5 . .1 .5 \mathrm{~m}$ with a fixed $p_{\min }=10$ was chosen, which were determined to be reasonable values in an automotive setting.

\section{Overall Assessment Method}

To determine the optimal parameter set for the $K N N$ $D B S C A N, 61$ radar measurements, containing a wide variety of driving maneuvers, like ac- and decelerating, left and right turning, lateral driving as well as in line with the sensor, forward and reversed movement, were evaluated. The score $s_{\mathscr{K}}(k, \alpha)$ for every parameter configuration was calculated and summed up over the measurements. The maximum score $s_{\mathscr{K} \text {, max }}$ was determined and tested for a confidence area $A_{\mathrm{c}}$ relative to the $k \times \alpha$ area where the score does not fall below $75 \%$ of $s_{\mathscr{K} \text {,max }}$. This serves as a measurement of operational stability due to fluctuations of data characteristics.

\section{RESULTS}

\section{A. Parameter Sweep}

The results of the parameter sweep conducted are presented in Tab. I. As can be seen, the rectangular kernel reaches the highest score of $s_{\mathscr{K}, \max }=12.5$ at $k=130$ and $\alpha=1.5$. Additionally, it is able to achieve similar results in a confidence area of $A_{\mathrm{c}}=28 \%$ of the parameter space around its optimal parameter set and thus can be considered as very stable. The DBSCAN result, which is not listed in the table, reaches a score of $s_{\text {DBSCAN }, \max }=11.8$ for $\epsilon=1.5 \mathrm{~m}$.

TABLE I

SCORING TABLE FOR KNN-DBSCAN

\begin{tabular}{r|lr} 
Kernel $\mathscr{K}$ & $s_{\mathscr{K}, \max }$ & $A_{\mathrm{c}}$ \\
\hline Gaussian & $10.0(@ k=70, \alpha=0.9)$ & $6 \%$ \\
Rectangular & $\mathbf{1 2 . 5}(@=130, \alpha=1.5)$ & $\mathbf{2 8 \%}$ \\
Triangular & $9.2(@ k=200, \alpha=1.0)$ & $13 \%$ \\
Epanechnikov & $9.8(@ k=120, \alpha=0.7)$ & $6 \%$
\end{tabular}

Figures 4 (a) and (b) show the clustering result, as well as the contour estimation on basis of a oriented bounding box with a $K N N-D B S C A N$ clustering using the rectangular kernel and $k=130$ and $\alpha=1.5$. The resulting errors $e_{\text {type }}$ with respect to the ground truth for both scenarios are listed in Tab. II.

TABLE II

CONTOUR ESTIMATION ERRORS

\begin{tabular}{c|cccc} 
& $e_{\text {area }}\left[\mathrm{m}^{2}\right]$ & $e_{\text {lenght }}[\mathrm{m}]$ & $e_{\text {width }}[\mathrm{m}]$ & $e_{\text {yaw }}\left[{ }^{\circ}\right]$ \\
\hline $\mathrm{A}$ & 2.3 & 0.12 & 0.53 & 0.94 \\
$\mathrm{~B}$ & 3.7 & 0.76 & 0.55 & 4.13
\end{tabular}

\section{B. Comparison DBSCAN vs. KNN-DBSCAN}

Regarding the score, the $K N N-D B S C A N$ is around the same level as the DBSCAN in the case of clearly shaped out reflection points like in scenario $\mathrm{A}$, where it delivers the same output in regards to the resulting contour estimation. However, in challenging situations, like scenario $\mathrm{B}, K N N-D B S C A N$ is able to find the cluster within the ground truth, despite the very inhomogeneous distribution. DBSCAN on the other hand fails to find any usable cluster in this situation.

However, this advantage comes at higher computational cost, with the generic implementation being around 5 times slower than DBSCAN depending on the number of target points and mostly due to the distance distribution calculation.

\section{CONCLUSION}

The adaptive clustering algorithm $K N N-D B S C A N$ was examined for its suitability in radar-based contour estimation applications. It shows promising results, especially in challenging conditions of very inhomogeneous reflection point distribution, where it surpasses the more basic DBSCAN. Simultaneously, it delivers the same robustness in scenarios with favorable reflection point behaviour. Due to the unique characteristics of radar data, a different approach of the distance examination step than originally proposed is needed.

\section{REFERENCES}

[1] E. Schubert, F. Meinl, M. Kunert, and W. Menzel, "High Resolution Automotive Radar Measurements of Vulnerable Road Users - Pedestrians \& Cyclists," in IEEE MTT-S International Conference on Microwaves for Intelligent Mobility (ICMIM), Apr. 2015, pp. 1-4.

[2] F. Roos, D. Kellner, J. Klappstein, J. Dickmann, K. Dietmayer, K. MullerGlaser, and C. Waldschmidt, "Estimation of the Orientation of Vehicles in High-Resolution Radar Images," in IEEE MTT-S International Conference on Microwaves for Intelligent Mobility (ICMIM), Apr. 2015, pp. $1-4$.

[3] M. Ester, H.-P. Kriegel, J. Sander, and X. Xu, "A Density-Based Algorithm for Discovering Clusters in Large Spatial Databases with Noise." in $K d d$, vol. 96, 1996, pp. 226-231.

[4] X. Yu, D. Zhou, and Y. Zhou, "A New Clustering Algorithm Based on Distance and Density," in International Conference on Services Systems and Services Management. Proceedings of ICSSSM, vol. 2, Jun. 2005 pp. 1016-1021.

[5] J. Jacques, C. Jung, and S. Musse, "Head-Shoulder Human Contour Estimation in Still Images," in IEEE International Conference on Image Processing (ICIP), Oct. 2014, pp. 278-282.

[6] S. Wender and K. Dietmayer, "3D Vehicle Detection Using a Laser Scanner and a Video Camera," IET Intelligent Transport Systems, vol. 2, pp. 105-112, Jun. 2008.

[7] R. Ratajczak, M. Domanski, and K. Wegner, "Vehicle Size Estimation from Stereoscopic Video," in 19th International Conference on Systems, Signals and Image Processing (IWSSIP), Apr. 2012, pp. 405-408.

[8] J. Sander, M. Ester, H.-P. Kriegel, and X. Xu, "Density-Based Clustering in Spatial Databases: The Algorithm GDBSCAN and Its Applications," Data Min. Knowl. Discov., vol. 2, no. 2, pp. 169-194, Jun. 1998. 